\title{
Generic Subsets in Spaces of Measures and Singular Continuous Spectrum
}

\author{
Daniel Lenz and Peter Stollmann \\ Fakultät für Mathematik, Technische Universität, 09107 Chemnitz, Germany \\ p.stollmann@mathematik.tu-chemnitz.de \\ d.lenz@@mathematik.tu-chemnitz.de
}

\begin{abstract}
We discuss recent results of ours showing that geometric disorder leads to some purely singularly continuous spectrum generically. This is based on a slight extension of Simons Wonderland theorem. Our approach to this theorem relies on the study of generic subsets of certain spaces of measures. In this article, we elaborate on this purely measure theoretic basis of our approach.
\end{abstract}

Key words: Random Schrödinger operators, Delone sets, spaces of measures, 81Q10,35J10,82B44, 28A33,28C15

\section{Introduction}

In this article we review our work [8] and elaborate on certain measure theoretic parts of it. The starting point of [8] is the study of operators

$$
H_{\omega}:=-\Delta+\sum_{x \in \omega} v(\cdot-x)
$$

on $L^{2}\left(\mathbb{R}^{d}\right)$, for suitable functions $v$ and certain uniformly discrete subsets $\omega$ of $\mathbb{R}^{d}$ called Delone sets. Such operators arise in the study of disordered solids. More precisely, they can be thought to model geometric disorder. It is shown that these operators have a purely singularly continuous spectral component generically. Here, generic refers to a topology on the set of all Delone sets (see below for details).

The abstract operator theoretic tool behind our reasoning is a slight strengthening of a result due to B. Simon called the "Wonderland theorem" [11]. Our method of proving this is different from Simons. It consists of two steps. We first prove that certain subsets of spaces of measures are generic. This generalizes the corresponding results of Simon [11] and Zamfirescu $[13]$ for $\mathbb{R}$ to rather general measure spaces. In the second step, the Wonderland theorem follows by considering spectral measures as continuous maps from the space of selfadjoint operators to measures on the real line.

Given our version of the Wonderland theorem, our result on generic singularly continuous spectrum follows from geometric considerations. There, we approximate arbitrary $\omega$ by essentially periodic ones in various ways.

D. Lenz and P. Stollmann: Generic Subsets in Spaces of Measures and Singular Continuous Spectrum, Lect. Notes Phys. 690, 333-341 (2006) 
In the present article, we particularly focus on the measure theoretic side of things. This is discussed in the next two sections. In particular, Sect. 3 elaborates on the last part of Sect. 2 of [8] and presents a generalization of Corollary 2.8 given there.

The subsequent discussion of the Wonderland theorem in Sect. 4 and the application to geometric disorder in Sect. 5 then follows [8] quite closely.

\section{Generic Subsets in Spaces of Measures}

We will be concerned with subsets of the set of positive, regular Borel measures $\mathcal{M}_{+}(S)$ on some locally compact, $\sigma$-compact, separable metric space $S$. The closed ball around $x \in S$ with radius $r$ is denoted by $B_{r}(x)$. The space $\mathcal{M}_{+}(S)$ is endowed with the weak topology from $C_{c}(S)$, also called the vague topology. We refer the reader to [3] for standard results concerning the space of measures. We will use in particular that the vague topology is metrizable such that $\mathcal{M}_{+}(S)$ becomes a complete metric space. Thus, the Baire category theorem becomes applicable. For the application to spectral theory, $S$ is just an open subset $U$ of the real line.

We call a measure $\mu \in \mathcal{M}_{+}(S)$ continuous if its atomic or pure point part vanishes, i.e. if $\mu(\{x\})=0$ for every $x \in S$. (We prefer the former terminology in the abstract framework and the latter for measures on the real line.) The set of all continuous measures on $S$ is denoted by $\mathcal{M}_{c}(S)$. A measure $\mu$ is called a point measure if there exists a countable set $Y$ in $X$ with $\mu(X \backslash Y)=0$. The set of all point measures on $S$ is denoted by $\mathcal{M}_{p}(S)$. This set is dense in $\mathcal{M}_{+}(S)$. Two measures are said to be mutually singular, $\mu \perp \nu$, if there exists a set $C \subset S$ such that $\mu(C)=0=\nu(S \backslash C)$. The set of all measures on $S$ which are singular with respect to a measure $\lambda$ is denoted by $\mathcal{M}_{\lambda, \operatorname{sing}}(S)$.

Our main generic result on spaces of measures reads as follows.

Theorem 1. Let $S$ be a locally compact, $\sigma$-compact, separable complete metric space. Then, the following holds:

(1) The set $\mathcal{M}_{c}(S)$ is a $G_{\delta}$-set in $\mathcal{M}_{+}(S)$.

(2) For any $\lambda \in \mathcal{M}_{+}(S)$, the set $\mathcal{M}_{\lambda, \text { sing }}(S)$ is a $G_{\delta}$-set in $\mathcal{M}_{+}(S)$.

(3) For any closed $F \subset S$ the set $\left\{\mu \in \mathcal{M}_{+}(S) \mid F \subset \operatorname{supp}(\mu)\right\}$ is a $G_{\delta}$-set in $\mathcal{M}_{+}(S)$.

\section{Singular Continuity of Measures}

As a first application of Theorem 1, we obtain a result on genericity of singular continuous spectrum. This generalizes the corresponding results of Simon [11] and Zamfirescu $[13,14]$ for measures on the real line. The first result of this 
section elaborates on Corollary 2.8 of $[8]$ and generalizes the result mentioned there. The second result seems to be new.

Theorem 2. Let $S$ be be locally compact, $\sigma$-compact, separable complete metric space. Let $\lambda$ be a continuous measure on $S$ with $\operatorname{supp} \lambda=S$. Then,

$$
\left\{\mu \in \mathcal{M}_{+}(S) \mid \mu \text { continuous and } \mu \perp \lambda\right\}
$$

is a dense $G_{\delta}$-set in $\mathcal{M}_{+}(S)$.

Proof. The set in question is the intersection of $\mathcal{M}_{c}(S)$ and $\mathcal{M}_{\lambda, s i n g}(S)$. By Theorem $1, \mathcal{M}_{c}(S)$ and $\mathcal{M}_{\lambda, \text { sing }}(S)$ are $G_{\delta}$-sets. Thus, by the Baire category theorem, it remains to show that $\mathcal{M}_{c}(S)$ and $\mathcal{M}_{\lambda, \text { sing }}(S)$ are dense. Denseness of these two sets follows from continuity of $\lambda$ : By continuity of $\lambda, \mathcal{M}_{\lambda, s i n g}(S)$ contains all point measures and is therefore dense. Also, by continuity of $\lambda$, $\mathcal{M}_{c}(S)$ is dense (see e.g. (ii) of the next theorem).

The previous theorem assumes the existence of a suitable measure $\lambda$. Such a $\lambda$ does not always exist. Instead the following is valid.

Theorem 3. Let $S$ be a locally compact $\sigma$-compact separable complete metric space. Then, the following assertions are equivalent:

(i) There exists a continuous measure $\lambda$ on $S$ with $\operatorname{supp} \lambda=S$.

(ii) The set $\mathcal{M}_{c}(S)$ is dense in $\mathcal{M}_{+}(S)$.

(iii) $S$ has no isolated points.

Proof. (i) $\Longrightarrow$ (ii): Let $x \in S$ be arbitrary. Then, for any $n \in \mathbb{N}$, the measure $\mu_{n}$ with

$$
\mu_{n}:=\left.\lambda\left(B_{1 / n}(x)\right)^{-1} \lambda\right|_{B_{1 / n}(x)}
$$

is continuous. Moreover, the sequence $\left(\mu_{n}\right)$ converges towards the unit point mass at $x$. Thus, every point measure can be approximated by continuous measures. As the point measures are dense, so are the continuous measures.

(ii) $\Longrightarrow$ (iii): This is clear.

(iii) $\Longrightarrow$ (i): We start with the following intermediate result:

Claim. For each closed ball $B$ in $S$ with positive radius, there exists a continuous probability measure $\mu_{B}$ whose support is contained in $B$.

Proof of claim. Denote the metric on $S$ by $d$. Let $x \in S$ and $r>0$ with $B=B_{r}(x)$ be given. As $S$ is locally compact, we can assume without loss of generality that $B$ is compact.

Define $\delta_{0}:=r / 4$ and consider $B_{\delta_{0}}(x)$. By (iii), $B_{\delta_{0}}(x)$ contains two different points $x_{1}^{(1)}$ and $x_{2}^{(1)}$. Set $\delta_{1}:=1 / 4 d\left(x_{1}^{1}, x_{2}^{(1)}\right)$ and consider $B_{\delta_{1}}\left(x_{2}^{(1)}\right)$ and $B_{\delta_{1}}\left(x_{2}^{(1)}\right)$. These balls are disjoint and by (ii) each contains two different points, i.e. there exist $x_{1}^{(2)}, x_{2}^{(2)}$ in $B_{\delta_{1}}\left(x_{1}^{(1)}\right)$ and $x_{3}^{(2)}, x_{4}^{(2)}$ in $B_{\delta_{1}}\left(x_{2}^{(1)}\right)$. 
Set $\delta_{2}:=1 / 4 \min \left\{d\left(x_{i}^{(2)}, x_{j}^{(2)}\right): i, j=1, \ldots, 4\right\}$. Then, the balls $B_{\delta_{2}}\left(x_{j}^{(2)}\right)$, $j=1, \ldots, 4$, are disjoint. Proceeding inductively, we can construct for each $n \in \mathbb{N}$ a $\delta_{n}>0$ and a set

$$
X_{n}:=\left\{x_{1}^{(n)}, \ldots, x_{2^{n}}^{(n)}\right\}
$$

with $2^{n}$ elements such that

$$
\delta_{n+1} \leq \frac{\delta_{n}}{2}, X_{n} \subset B,
$$

and for each $y \in B$, and $n \geq k$, the ball $B_{\delta_{k}}(y)$ contains at most $2^{n-k}$ points of $X_{n}$. Now, consider, for each $n \in \mathbb{N}$, the measure

$$
\mu_{n}:=\frac{1}{2^{n}} \sum_{j=1}^{2^{n}} \delta_{x_{j}^{(n)}} .
$$

Then each $\mu_{n}$ is a probability measure supported in $B$ and

$$
\mu_{n}\left(B_{\delta_{k}}(y)\right) \leq \frac{1}{2^{n}} \times 2^{n-k}=2^{-k}
$$

for every $k \leq n$ and $y \in S$. By the Theorem of Banach/Alaoglu, the sequence $\left(\mu_{n}\right)$ has a converging subsequence. Thus, without loss of generality, we can assume that the sequence itself converges to a measure $\mu$. As each $\mu_{n}$ is a probability measure supported in $B$ and $B$ is compact, $\mu$ is a probability measure supported in $B$ as well. Moreover, by (1), $\mu$ is continuous. This finishes the proof of the claim.

Let $D$ be a countable dense subset of $S$. For each $x \in D$ and $n \in \mathbb{N}$, we can find a continuous probability measure $\mu_{n, x}:=\mu_{B_{\frac{1}{n}}(x)}$ supported in $B_{\frac{1}{n}}(x)$ according to the claim. Now, choose numbers $c_{n, x}>0, x \in D$ and $n \in \mathbb{n} \in \mathbb{N}$, with

$$
\sum_{n \in \mathbb{N}, x \in D} c_{n, x}<\infty
$$

Then,

$$
\lambda:=\sum_{n \in \mathbb{N}, x \in D} c_{n, x} \mu_{n, x}
$$

is a continuous measure whose support contains all $x \in D$. As $D$ is dense, the support of $\lambda$ equals $S$ and (i) follows.

\section{Selfadjoint Operators and the Wonderland Theorem}

In this section, we discuss a consequence of Theorem 2 on generic appearance of a purely singular continuous component in the spectrum for certain Hamiltonians. 
This provides a slight strengthening of Simon's "Wonderland Theorem" from [11]. The main point of our discussion, however, is not this strengthening but rather the new proof we provide.

In order to formulate our result, let us introduce the following notation:

For a fixed separable Hilbert space $\mathcal{H}$ consider the space $\mathcal{S}=\mathcal{S}(\mathcal{H})$ of self-adjoint operators in $\mathcal{H}$. For $\xi \in \mathcal{H}$ and $A \in \mathcal{S}$ let the spectral measure $\rho_{\xi}^{A}$ be defined by

$$
\rho_{\xi}^{A}(\varphi):=\langle\xi, \varphi(A) \xi\rangle
$$

for each continuous $\varphi$ on $\mathbb{R}$ with compact support.

We endow $\mathcal{S}$ with the strong resolvent topology $\tau_{\text {srs }}$, the weakest topology for which all the mappings

$$
\mathcal{S} \rightarrow \mathbb{C}, A \mapsto(A+i)^{-1} \xi \quad(\xi \in \mathcal{H})
$$

are continuous. Therefore, a sequence $\left(A_{n}\right)$ converges to $A$ w.r.t. $\tau_{s r s}$ if and only if

$$
\left(A_{n}+i\right)^{-1} \xi \rightarrow(A+i)^{-1} \xi
$$

for all $\xi \in \mathcal{H}$. Thus, for each $\xi \in \mathcal{H}$, the mapping

$$
\rho_{\xi}: \mathcal{S} \longrightarrow \mathcal{M}(\mathbb{R}), \quad A \mapsto \rho_{\xi}^{A},
$$

is continuous.

The spectral subspaces of $A \in \mathcal{S}$ are defined by

$$
\begin{aligned}
\mathcal{H}_{a c}(A) & =\left\{\xi \in \mathcal{H} \mid \rho_{\xi}^{A} \text { is absolutely continuous }\right\} \\
\mathcal{H}_{s c}(A) & =\left\{\xi \in \mathcal{H} \mid \rho_{\xi}^{A} \text { is singular continuous }\right\} \\
\mathcal{H}_{c}(A) & =\left\{\xi \in \mathcal{H} \mid \rho_{\xi}^{A} \text { is continuous }\right\} \\
\mathcal{H}_{p p}(A) & =\mathcal{H}_{c}(A)^{\perp}, \mathcal{H}_{s}(A)=\mathcal{H}_{a c}(A)^{\perp} .
\end{aligned}
$$

These subspaces are closed and invariant under $A . \mathcal{H}_{p p}(A)$ is the closed linear hull of the eigenvectors of $A$. Recall that the spectra $\sigma_{*}(A)$ are just the spectra of $A$ restricted to $\mathcal{H}_{*}(A)$.

Theorem 4. Let $(X, \rho)$ be a complete metric space and $H:(X, \rho) \rightarrow\left(\mathcal{S}, \tau_{\text {srs }}\right)$ a continuous mapping. Assume that, for an open set $U \subset \mathbb{R}$,

(1) the set $\left\{x \in X \mid \sigma_{p p}(H(x)) \cap U=\emptyset\right\}$ is dense in $X$,

(2) the set $\left\{x \in X \mid \sigma_{a c}(H(x)) \cap U=\emptyset\right\}$ is dense in $X$,

(3) the set $\{x \in X \mid U \subset \sigma(H(x))\}$ is dense in $X$.

Then, the set

$$
\left\{x \in X \mid U \subset \sigma(H(x)), \sigma_{a c}(H(x)) \cap U=\emptyset, \sigma_{p p}(H(x)) \cap U=\emptyset\right\}
$$

is a dense $G_{\delta}$-set in $X$. 
A proof can be sketched as follows (see [8] for details): By assumption $H$ is continuous. Furthermore, the restriction $r_{U}: \mathcal{M}(\mathbb{R}) \longrightarrow \mathcal{M}(U),\left.\mu \mapsto \mu\right|_{U}$, can easily be seen to be continuous as well. Finally, as discussed above, for each $\xi \in \mathcal{H}$, the map $\rho_{\xi}$ is continuous. Thus, the composition

$$
\mu_{\xi}:=r_{U} \circ \rho_{\xi} \circ H: X \longrightarrow \mathcal{M}(U),\left.x \mapsto \rho_{\xi}^{H(x)}\right|_{U},
$$

is a continuous map. Thus, the inverse image of a $G_{\delta}$-set in $\mathcal{M}(U)$ under $\mu_{\xi}$ is a $G_{\delta}$-set in $X$. Thus, by Theorem 1, the sets $\left\{x \in S: \mu_{\xi}(x)\right.$ is continuous $\}$, $\left\{x \in S: \mu_{\xi}(x)\right.$ is singular w.r.t. Lebesgue measure $\}$ and $\left\{x \in S: \operatorname{supp} \mu_{\xi}(x)\right.$ contains $U\}$ are all $G_{\delta}$-sets. Moreover, by assumption they are dense. Thus, their intersection is a dense $G_{\delta}$-set by the Baire category theorem. One more intersection over a countable dense subset of $\xi \in \mathcal{H}$ yields the desired result. This finishes the proof of the theorem.

We say that the spectrum of $A \in \mathcal{S}$ is pure point (purely absolutely continuous, purely singularly continuous) on $U \subset \mathbb{R}$ if the restrictions $\rho_{\xi}^{A} \mid U$ have the corresponding properties. Of course, if $A$ has pure point (purely absolutely continuous) spectrum on $U$ it does not have any absolutely continuous (pure point) spectrum on $U$. As in [11], the theorem has then the following immediate but remarkable corollary.

Corollary 1. Let $(X, \rho)$ be a complete metric space and $H:(X, \rho) \rightarrow$ $\left(\mathcal{S}, \tau_{\text {srs }}\right)$ a continuous mapping. Assume that, for an open set $U \subset \mathbb{R}$,

(1) the set $\{x \in X \mid H(x)$ has pure point spectrum in $U\}$ is dense in $X$,

(2) the set $\{x \in X \mid H(x)$ has purely absolutely continuous spectrum in $U\}$ is dense in $X$,

(3) the set $\{x \in X \mid U \subset \sigma(H(x))\}$ is dense in $X$.

Then, the set

$$
\left\{x \in X \mid U \subset \sigma(H(x)), \sigma_{a c}(H(x)) \cap U=\emptyset, \sigma_{p p}(H(x)) \cap U=\emptyset\right\}
$$

is a dense $G_{\delta}$-set in $X$.

\section{Operators Associated to Delone Sets}

In this section we discuss an application of Theorem 4 to geometric disorder.

We start by recalling the necessary notation. A key notion is the notion of Delone set, named after B.N. Delone (Delaunay), [6]. The Euclidean norm on $\mathbb{R}^{d}$ is denoted by $\|\cdot\|$. We replace $\mathrm{B}$ by $\mathrm{U}$ to denote open balls.

Definition 1. A set $\omega \subset \mathbb{R}^{d}$ is called an $(r, R)$-set if

- $\forall x, y \in \omega, x \neq y:\|x-y\|>r$,

- $\forall p \in \mathbb{R}^{d} \exists x \in \omega:\|x-p\| \leq R$. 
By $\mathbb{D}_{r, R}\left(\mathbb{R}^{d}\right)=\mathbb{D}_{r, R}$ we denote the set of all $(r, R)$-sets. We say that $\omega \subset \mathbb{R}^{d}$ is a Delone set, if it is an $(r, R)$-set for some $0<r \leq R$ so that $\mathbb{D}\left(\mathbb{R}^{d}\right)=$ $\mathbb{D}=\bigcup_{0<r \leq R} \mathbb{D}_{r, R}\left(\mathbb{R}^{d}\right)$ is the set of all Delone sets.

Delone sets turn out to be quite useful in the description of quasicrystals and more general aperiodic solids; see also [4], where the relation to discrete operators is discussed. In fact, if we regard an infinitely extended solid whose ions are assumed to be fixed, then the positions are naturally distributed according to the points of a Delone set. Fixing an effective potential $v$ for all the ions this leads us to consider the Hamiltonian

$$
H(\omega):=-\Delta+\sum_{x \in \omega} v(\cdot-x) \text { in } \mathbb{R}^{d},
$$

where $\omega \in \mathbb{D}$. Let us assume, for simplicity that $v$ is bounded, measurable and compactly supported.

In order to apply our analysis above, we need to introduce a suitable topology on $\mathbb{D}$. This can be done in several ways, cf. $[4,7]$. The emerging topology is called the natural topology. It defines a compact, complete metrizable topology on the set of all closed subsets of $\mathbb{R}^{d}$ for which $\mathbb{D}_{r, R}\left(\mathbb{R}^{d}\right)$ is a compact, complete space. We refrain from giving the exact definition of this topology here and refer to the cited literature. Instead we note the following lemma, which describes convergence w.r.t the natural topology.

Lemma 1. A sequence $\left(\omega_{n}\right)$ of Delone sets converges to $\omega \in \mathbb{D}$ in the natural topology if and only if there exists for any $l>0$ an $L>l$ such that the $\omega_{n} \cap U_{L}(0)$ converge to $\omega \cap U_{L}(0)$ with respect to the Hausdorff distance as $n \rightarrow \infty$.

Given the lemma, it is not hard to see that the map

$$
H: \mathbb{D}_{r, R}\left(\mathbb{R}^{d}\right) \longrightarrow \mathcal{S}\left(L^{2}\left(\left(\mathbb{R}^{d}\right)\right), \omega \mapsto H(\omega),\right.
$$

is continuous.

Finally, we recall that a Delone set $\gamma$ on $\mathbb{R}^{d}$ is called crystallographic if the set of its periods

$$
\operatorname{Per}(\gamma):=\left\{t \in \mathbb{R}^{d}: t+\gamma=\gamma\right\}
$$

is a lattice of full rank in $\mathbb{R}^{d}$. Now our result on generic singularly continuous spectrum can be stated as follows.

Theorem 5. Let $r, R>0$ with $2 r \leq R$ and $v$ be given such that there exist crystallographic $\gamma, \tilde{\gamma} \in \mathbb{D}_{r, R}$ with $\sigma(H(\gamma)) \neq \sigma(H(\tilde{\gamma}))$. Then

$$
U:=\left(\sigma(H(\gamma))^{\circ} \backslash \sigma(H(\tilde{\gamma}))\right) \cup\left(\sigma(H(\tilde{\gamma}))^{\circ} \backslash \sigma(H(\gamma))\right)
$$

is nonempty and there exists a dense $G_{\delta}$-set $\Omega_{s c} \subset \mathbb{D}_{r, R}$ such that for every $\omega \in \Omega_{\text {sc }}$ the spectrum of $H(\omega)$ contains $U$ and is purely singular continuous in $U$. 
A proof can be sketched as follows (see [8] for details):

We let $U_{1}:=\sigma(H(\gamma))^{\circ} \backslash \sigma(H(\tilde{\gamma}))$ and $U_{2}:=\sigma(H(\tilde{\gamma}))^{\circ} \backslash \sigma(H(\gamma))$. Since $\gamma, \tilde{\gamma}$ are crystallographic, the corresponding operators are periodic and their spectra are consequently purely absolutely continuous and consist of a union of closed intervals with only finitely many gaps in every compact subset of the reals. Hence, under the assumption of the theorem $U_{1}$ or $U_{2}$ is nonempty. Thus, $U$ is nonempty.

We now consider the case that $U_{1}$ is nonempty. We will verify conditions (1)-(3) from Theorem 4.

Ad (1): Fix $\omega \in \mathbb{D}_{r, R}$. For $n \in \mathbb{N}$ consider $\nu_{n}:=\omega \cap Q(n)$. We can then periodically extend $\nu_{n}$, i.e. we find crystallographic $\omega_{n}$ in $\mathbb{D}_{r, R}$ with $\omega_{n} \cap Q(n)=\nu_{n}$. For given $L>0$ we get that $\omega_{n} \cap U_{L}(0)=\omega \cap U_{L}(0)$ if $n$ is large enough. Therefore, by Lemma 1 , we find that $\omega_{n} \rightarrow \omega$ with respect to the natural topology. On the other hand, $\sigma_{p p}\left(H\left(\omega_{n}\right)\right)=\emptyset$ since the potential of $H\left(\omega_{n}\right)$ is periodic. Consequently,

$$
\left\{\omega \in \mathbb{D}_{r, R} \mid \sigma_{p p}(H(\omega)) \cap U_{1}=\emptyset\right\}
$$

is dense in $\mathbb{D}_{r, R}$.

Ad (2): We have to show denseness of $\omega$ for which $\sigma_{a c}(H(\omega)) \cap U_{1}=\emptyset$. Thus, fix $\omega \in \mathbb{D}_{r, R}$. Then, we can construct $\omega_{n}$ which agree with $\omega$ around zero and with $\widetilde{\gamma}$ away from zero. More precisely, for $n \in \mathbb{N}$ large enough, find $\omega_{n} \in \mathbb{D}_{r, R}$ such that

$$
\omega_{n} \cap U_{n}(0)=\omega \cap U_{n}(0) \text { and } \omega_{n} \cap U_{2 n}(0)^{c}=\widetilde{\gamma} \cap U_{2 n}(0)^{c} .
$$

In virtue of the last property, $H\left(\omega_{n}\right)$ and $H(\widetilde{\gamma})$ only differ by a compactly supported, bounded potential, so that $\sigma_{a c}\left(H\left(\omega_{n}\right)\right)=\sigma_{a c}(H(\widetilde{\gamma})) \subset U_{1}^{c}$. Again, $\omega_{n} \rightarrow \omega$ yields condition (2) of Theorem 4 .

Ad (3): This can be checked with a similar argument as (2), this time with $\tilde{\gamma}$ instead of $\gamma$. More precisely, fix $\omega \in \mathbb{D}_{r, R}$. For $n \in \mathbb{N}$ large enough, we find $\omega_{n} \in \mathbb{D}_{r, R}$ such that

$$
\omega_{n} \cap U_{n}(0)=\omega \cap U_{n}(0) \text { and } \omega_{n} \cap U_{2 n}(0)^{c}=\gamma \cap U_{2 n}(0)^{c} .
$$

In virtue of the last property, $H\left(\omega_{n}\right)$ and $H(\gamma)$ only differ by a compactly supported, bounded potential, so that $\sigma_{a c}\left(H\left(\omega_{n}\right)\right)=\sigma_{a c}(H(\gamma)) \supset U_{1}$. By $\omega_{n} \rightarrow \omega$, we obtain (3) of Theorem 4 .

As a consequence of these considerations, Theorem 4 gives that

$$
\left\{\omega \in \mathbb{D}_{r, R} \mid \sigma_{p p}(H(\omega)) \cap U_{1}=\emptyset, \sigma_{a c}(H(\omega)) \cap U_{1}=\emptyset, U_{1} \subset \sigma(H(\omega))\right\}
$$

is a dense $G_{\delta}$-set if $U_{1}$ is not empty. An analogous argument shows the same statement with $U_{2}$ instead of $U_{1}$. This proves the assertion if only one of the $U_{i}, i=1,2$, is not empty. Otherwise, the assertion follows after intersecting the two dense $G_{\delta}$ 's. This finishes the proof of the theorem. 


\section{Acknowledgment}

The research presented above has been partly supported by the DFG. Useful comments of J. Voigt are gratefully acknowledged. We would also like to take this opportunity to thank the organizers of QMath9 for the stimulating and relaxed atmosphere.

\section{References}

1. M. Baake, R.V. Moody (eds), Directions in mathematical quasicrystals, CRM Monogr. Ser., 13, Amer. Math. Soc., Provicence, RI, 2000.

2. S. Banach, Über die Bairesche Kategorie gewisser Funktionenmengen. Studia Math. 3 (1931), 174-179.

3. H. Bauer, Maß- und Integrationstheorie, De Gruyter, Berlin, 1990.

4. J. Bellissard, D.J.L. Hermann, and M. Zarrouati, Hulls of Aperiodic Solids and Gap Labeling Theorem, In: [1], pp. 207-258.

5. D. Buschmann, G. Stolz, Two-parameter spectral averaging and localization for non-monotonic random Schrödinger operators, Trans. Amer. Math. Soc. 353 (2001), 635-653.

6. B. Delaunay [B.N. Delone], Sur la sphère vide, Izvestia Akad Nauk SSSR Otdel. Mat. Sov. Nauk. 7, 1934, pp. 793-800.

7. D. Lenz and P. Stollmann, Delone dynamical systems and associated random operators, Proceedings, Constanta (Romania), 2001, eds. J.-M. Combes et al., Theta Foundation, eprint: arXiv:math-ph/0202042.

8. D. Lenz and P. Stollmann, Generic sets in spaces of measures and generic singular continuous spectrum for Delone Hamiltonians, eprint: arXiv:math-ph/0410021, to appear in Duke Math. J..

9. S. Mazurkiewicz, Sur les fonctions non dérivables, Studia Math. 3 (1931), 92-94.

10. R.V. Moody (ed.), The mathematics of long-range aperiodic order. NATO Advanced Science Institutes Series C: Mathematical and Physical Sciences, 489. Kluwer Academic Publishers Group, Dordrecht, 1997.

11. B. Simon, Operators with singular continuous spectrum: I. General operators. Annals of Math. 141, 131-145 (1995).

12. J. Weidmann, Lineare Operatoren in Hilberträumen. Teil I: Grundlagen. B.G. Teubner, Stuttgart, 2000.

13. T. Zamfirescu, Most monotone functions are singular, Amer. Math. Monthly 88 (1981), 456-458.

14. T. Zamfirescu, Typical monotone continuous functions, Arch. Math. 42 (1984), $151-156$. 
